\title{
Diode Laser Cyclophotocoagulation in the Management of Pain and Intraocular Pressure Control in Patients with Terminal Glaucoma-Six Years' Experience
}

\author{
Silvestre Cruz ${ }^{1}$, Diogo Cabral' ${ }^{1}$, Filipe Braz ${ }^{1}$, Ana Vide Escada ${ }^{2}$, Jorge Godinho Ferreira ${ }^{1}$, \\ José Fernandes ${ }^{1}$, João Segurado ${ }^{1}$ \\ ${ }^{1}$ Instituto de Oftalmologia Dr. Gama Pinto \\ ${ }^{2}$ Centro de Responsabilidade de Oftalmologia do Hospital Garcia de Orta, Lisbon, Portugal \\ Email: Silvestre.dacruz@hotmail.com
}

How to cite this paper: Cruz, S., Cabral, D., Braz, F., Escada, A.V., Ferreira, J.G., Fernandes, J. and Segurado, J. (2017) Diode Laser Cyclophotocoagulation in the Management of Pain and Intraocular Pressure Control in Patients with Terminal Glaucoma-Six Years' Experience. Open Journal of Ophthalmology, 7, 88-94.

https://doi.org/10.4236/ojoph.2017.72013

Received: March 24, 2017

Accepted: May 15, 2017

Published: May 18, 2017

Copyright $\odot 2017$ by authors and Scientific Research Publishing Inc. This work is licensed under the Creative Commons Attribution International License (CC BY 4.0).

http://creativecommons.org/licenses/by/4.0/

\begin{abstract}
Objective: This study aims to describe the experience with diode laser cyclophotocoagulation in the control of intraocular pressure and resolution of pain in patients with refractory glaucoma. Methods: Retrospective study. 64 eyes of 60 patients who underwent cyclophotocoagulation between January 2008 and March 2014. Evaluation of the pre- and post-operative intraocular pressure, the control of ocular pain, the number of anti-glaucoma drugs used in preand post-intervention, the rate of complications and the success rate. Results: There was an overall success rate of $81.3 \%$, with 9 eyes needing a second intervention. There was a mean reduction of $52.6 \%$ of the preoperative mean intraocular pressure of 41.25 to $19.56 \mathrm{mmHg}$ at 12 months observation ( $\mathrm{p}<$ $0.001)$. There was also a statistically significant reduction in the number of anti-glaucoma drugs used from 3.19 to 2.01 per eye and the resolution of pain in $75 \%$ of patients. Conclusions: cyclophotocoagulation is an effective procedure, with an expected positive impact on quality of life of patients as a result from the decreased number of anti-glaucoma drugs, sustained intraocular pressure reduction and resolution of pain.
\end{abstract}

\section{Keywords}

Diode Laser Cyclophotocoagulation, Glaucoma, Painful Eye,

Intraocular Pressure, Quality of Life

\section{Introduction}

Refractory glaucoma-those not controlled by medical therapy and/or sur- 
gery-is a therapeutic challenge. Many of these patients, even with maximum tolerated dosages of medication maintain significant painful symptomology. In these cases, the technical resources available such as surgical filtration devices with shunts and/or antimetabolites have been used with good success rates [1] [2]. In cases where these techniques fail, the cyclodestructive techniques are an option [2].

Cyclodestructive procedures, unlike surgical filtrations, reduce the intra-ocular pressure (IOP) by reducing the production of aqueous humour due to the destruction of the ciliary body [1] [2] [3] [4]. Cyclophotocoagulation (CFC) is a cyclodestructive technique which may be performed via the transcleral, transpupillary or endoscopic routes. The trans-scleral approach is the most common and may be carried out using contact or non-contact techniques depending on whether the laser mechanism requires direct contact with the eye or not [2] [5]. The most frequently used equipments are the $1064 \mathrm{~nm} \mathrm{Nd:YAG} \mathrm{LASER} \mathrm{and} \mathrm{the}$ $810 \mathrm{~nm}$ diode LASER [1] [2] [3] [4]. O diode laser has the advantage of being portable and more accessibly priced than other lasers used for the same purpose [2]. Aside from that, in relation to the Nd:YAG LASER, the diode LASER presents better absorption by the uveal melanin, thus requiring less energy to obtain the desired photothermic alterations of the ciliary body [1] [3]. Histopathological studies have shown that the use of CFC effectively induces a coagulative necrosis of the pars plicata with slight extension to the pars plana in the treatment area, leading to the destruction of the ciliary body, reducing the production of aqueous humour and resulting diminished IOP [1] [5] [6] [7].

Due to the described risk of hypotonia and e phthisis bulbi, CFC has, usually, been reserved for patients with refractory glaucoma without indication for other surgical procedures or with whom previous surgeries have failed, patients without visual potential and those presenting with pain [1].

The aim of this research was to study if the realization of CFC would allow for: the reduction of intra-ocular pressure in the time intervals foreseen by our protocol, to diminish the number of anti-glaucoma drugs administered to patients and abolish the ocular pain experienced pre-procedure. Equally we aimed to evaluate the safety of the procedure with regards to temporary and serious adverse effects.

\subsection{Materials and Methods}

A retrospective observational study was carried out via the revision of the clinical files of patients submitted to CFC between January 2008 and March 2014. The inclusion criteria considered were: diagnosis of refractory glaucoma uncontrolled by other treatments and low visual acuity $(\mathrm{VA})(\mathrm{VA}<1 / 10)$. All procedures were performed with the same device o "IRIS Medical Oculight SLx" (UHS Inc, Mineapolis-USA) and 810nm G-Probe for trans-scleral application. The procedures were performed on an out-patient basis following peribulbar anaesthesia with $1 \%$ ropivicaine. With the patient in a supine position, the procedure was carried out based on the service protocol guidelines: the probe positioned $1.5 \mathrm{~mm}$ from the limbus and about 20 impacts at 270 degrees, sparing the 
meridian of 3 and 9 o'clock and the scleral regions of previous interventions, at a potency of 1500 to $2500 \mathrm{~mW}$ and 2 seconds duration. Data was collected at check ups at week 1, month 1, 3 months, 6 months and 12 months post-op. The following variables were examined: gender, age, primary diagnosis, ophthalmological history, IOP [applanation tonometry (Goldmann)], ocular pain (presente or absent), number of drugs (active ingredient), use of acetazolamide (yes or no), complications and post-operative occurrences and success (categorized yes or no in accordance with the criteria of the study). As criteria for success, post operative values $\leq 21 \mathrm{mmHg}$ in the case of IOP variation or a reduction $\geq 30 \%$ of the initial value and, in the case of pain evaluation, the resolution of previous painful symptomology.

In the cases in which there was a need to perform a second treatment with CFC, the measurements taken after the second treatment were those considered.

Patients with incomplete data were excluded. The data was processed using the IBM ${ }^{\oplus}$ SPSS $^{\circledast} 23.0$ (SPSS, Inc., 2016, Chicago, IL) programme and analysed to a level of confidence of $95 \%$.

\subsection{Results}

The files of 83 patients were analysed. All patients underwent CFC in the terminal glaucoma setting, but of these only 60 (64 eyes) complied with the inclusion criteria.

Our sample was characterized in accordance with the variables being studied prior to undergoing CFC (Table 1).

The ophthalmological histories were characterized in accordance with their frequency (Figure 1). From these we can highlight diabetic proliferative retinopathy with rubeosis iridis in 16 eyes (25\%).

The diagnosis was characterized in accordance to its frequency (Figure 2). From this we highlight that $48 \%$ of the eyes had an established diagnosis of neovascular glaucoma.

We studied the variation of IOP over time and after undergoing CFC (Figure 3) using the paired $t$ test (Table 2).

We saw that CFC reduced, on average, the IOP in a statistically significant manner $(\mathrm{p}<0.0001)$ regardless of the time interval, being that the difference in averages at 12 months surpassed the difference in averages in the other time intervals studied.

Table 1. Descriptive analysis.

\begin{tabular}{cc}
\hline & Average \\
\hline Age & $65 \pm 15$ \\
IOP before CFC (mmHg) & $41.25 \pm 8.67$ \\
Total drugs pre CFC & $3.2 \pm 0.7$ \\
Male & $\%$ \\
Ocular pain before CFC & $48 \%$ \\
\hline
\end{tabular}


Table 2. Statistical Analysis (IOPpre = Intra Ocular Pressure before diode laser cyclophotocoagulation; IOPpos = Intra Ocular Pressure after diode laser cyclophotocoagulation).

\begin{tabular}{|c|c|c|c|c|c|c|c|}
\hline & & \multicolumn{5}{|c|}{ Matched differences } & \multirow{3}{*}{$\begin{array}{c}\text { Sig. } \\
\text { (bilateral) }\end{array}$} \\
\hline & & \multirow{2}{*}{$\begin{array}{l}\text { Avarage } \\
(\mathrm{mmHg})\end{array}$} & \multirow{2}{*}{$\begin{array}{l}\text { Standard } \\
\text { deviation } \\
(\mathrm{mmHg})\end{array}$} & \multirow{2}{*}{$\begin{array}{c}\text { Mean } \\
\text { standard error }\end{array}$} & \multicolumn{2}{|c|}{ 95\% Difference confidence interval } & \\
\hline & & & & & $\begin{array}{l}\text { Inferior } \\
(\mathrm{mmHg})\end{array}$ & $\begin{array}{l}\text { Superior } \\
(\mathrm{mmHg})\end{array}$ & \\
\hline Par 1 & IOPpre-IOPpos 1 year & 21.689 & 10.756 & 1.377 & 18.934 & 24.443 & 0.000 \\
\hline Par 2 & IOPpre-IOPpos 6 months & 20.571 & 10.392 & 1.389 & 17.788 & 23.354 & 0.000 \\
\hline Par 3 & IOPpre-IOPpos 3 months & 18.095 & 9.884 & 1.245 & 15.606 & 20.585 & 0.000 \\
\hline Par 4 & IOPpre-IOPpos 1 month & 15.825 & 9.474 & 1.194 & 13.439 & 18.211 & 0.000 \\
\hline Par 5 & IOPpre-IOPpos 1 week & 12.175 & 9.825 & 1.301 & 9.568 & 14.782 & 0.000 \\
\hline
\end{tabular}

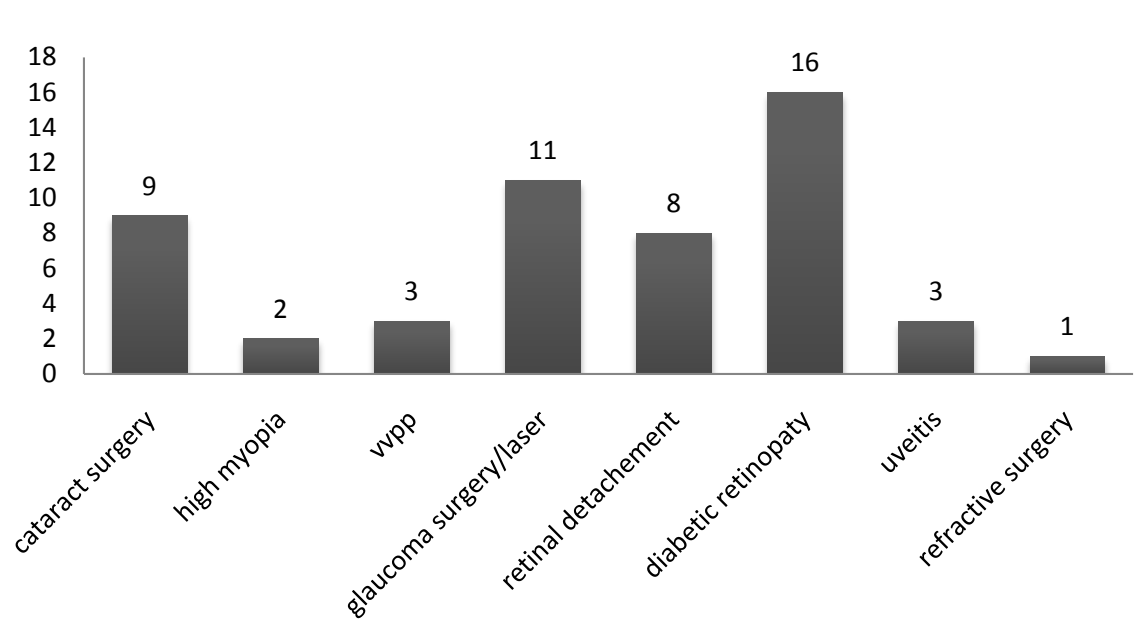

Figure 1. Relevant ophthalmological history (vvpp = vitrectomy via pars plana).

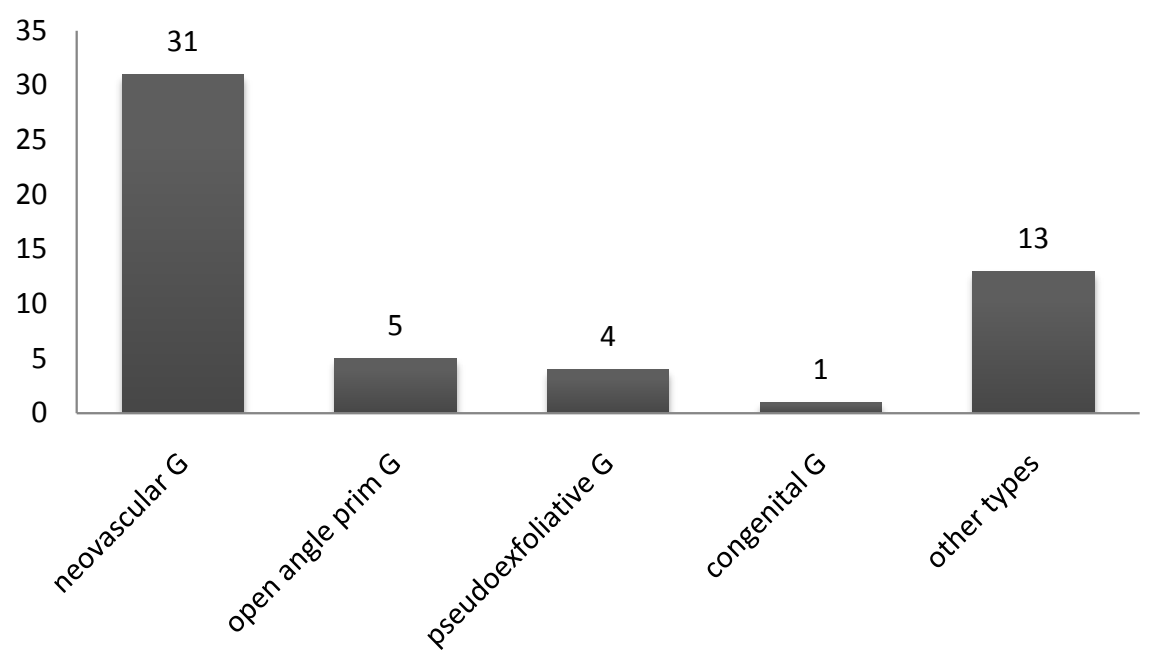

Figure 2. Glaucoma Type ( $\mathrm{G}$ = glaucoma; prim = primary).

The variation of the number of anti glaucoma drugs was studied using the paired $t$ test. The average number of drugs used pre-CFC was three $(3.2 \pm 0.7)$ and after undergoing CFC two $(2 \pm 0.8)$. The difference in averages was 1.2 (LC $95 \%[0.95-1.4], \mathrm{p}<0.0001)$. 


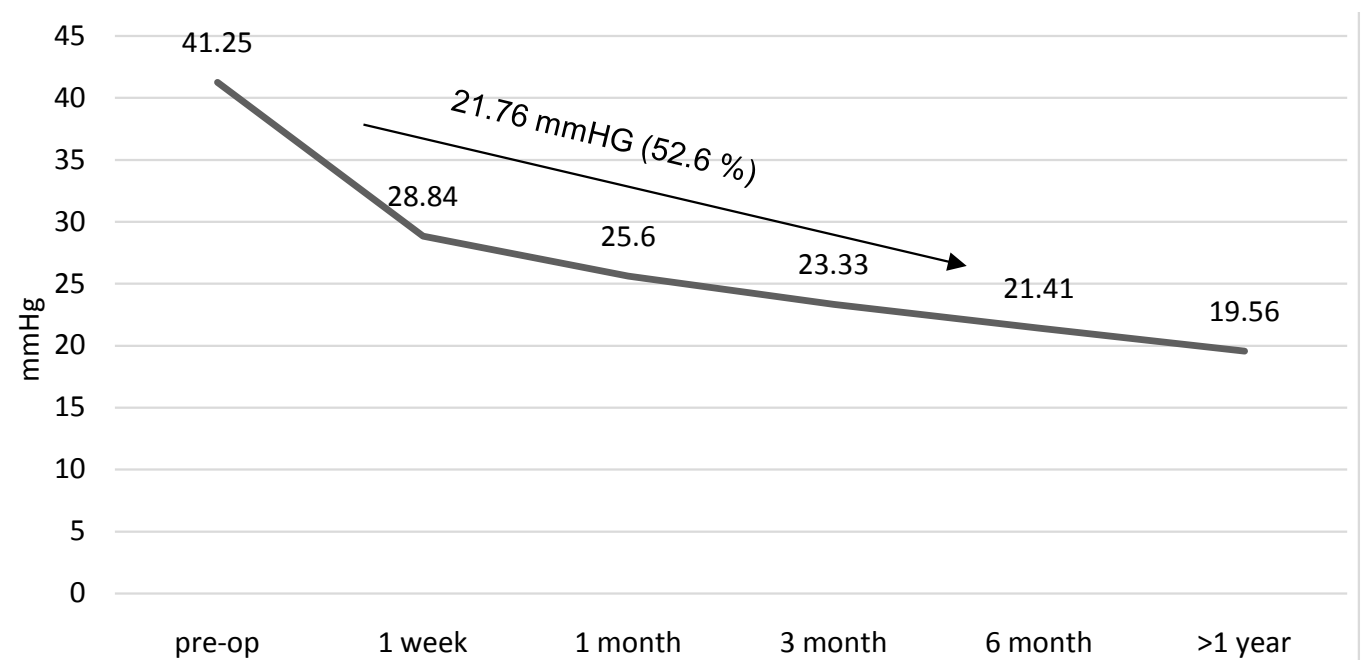

Figure 3. Evaluation of reduction of intraocular pressure $(\mathrm{mmHg})$ post treatment with cyclophotocoagulation (pre-op $=$ pre-operative).

In $70 \%$ of cases ( 45 eyes), there was a presence of painful symptomology. After undergoing $\mathrm{CFC}, 11$ eyes maintained complaints of pain which represents a $75 \%$ reduction of the same. This variation was analysed by the McNemar test, demonstrating a high level of statistical significance $(p<0.0001)$.

Regarding the analysis of the complications inherent in the procedure, six (9.3\%) major complications were observed: four cases of phthisis bulbi, two cases of seclusio pupillae and nine (14\%) minor complications: six cases of hyphaema of the anterior chamber (9\%), two cases of anterior segment inflammation (3\%) and one case of keratatic ulcer (1.5\%).

As regards the analysis of therapeutic success, in 46 eyes (72\%) therapeutic success was achieved after a single intervention and in nine eyes (14\%) a second intervention was with CFC was necessary (and success was achieved in six eyes (66\%). Thus a global number of successes was achieved (after one or two CFC sessions) in $81.3 \%$ cases (52 eyes).

\section{Discussion}

CFC with trans-scleral diode LASER has been used for over 15 years to treat patients with glaucoma which is refractory to pharmacological and surgical therapy, generally as a final attempt at therapeutic or palliative treatment to control IOP an/or painful ocular symptomology [1] [2] [3] [7] [8] and is still a viable option.

Our results corroborate those of other studies, in which CFC has been shown to be an effective and secure technique for the reduction of IOP in refractory glaucoma [1] [5] [8] [9]. A success rate of $81.3 \%$ of cases was identified, similar to the rates described by other authors [1] [2] [3] [8] [10]. We verified that CFC does diminish the IOP in a statistically significant manner after a cycloablative procedure in all the intervals studied ( $\mathrm{p}<0.0001)$. We found an average diminishing of $52.6 \%$ at 12 months post-procedure, similar to that reported by other authors [2] [3] [5] [9]. 
The number of anti-glaucoma drugs has been signalled as an indicator of the reduction in expenses, secondary effects and improvement in quality of life [9]. We found there to be an average reduction of one drug (CI 95\% [0.95 - 1.4], p < 0.0001) following the use of CFC.

As for the pain in glaucoma patients, the improvement in quality of life obtained by resolving the ocular pain and by reducing anti-glaucoma drugs it is even more significant when dealing with neovascular, post-traumatic, aphakic glaucoma or those caused by silicone oil where there is a great difficulty in resolving symptomology and controlling IOP by the conventional medical and surgical techniques [9]. In our study the resolution of painful symptomology was observed in $75 \%$ of patients ( $p<0.0001$ ). As this is one of the main complaints from patients suffering from refractory glaucoma in more advanced stages the value obtained highlights the importance of CFC in painful eyes as an alternative to more invasive methods. As for the evaluation of serious adverse effects of the procedure, we should refer the frequency of complications observed in our study (6\% of phthisis bulbi and $3 \%$ of seclusio pupillae) is identical to the frequencies observed in other studies [1] [2] [3] [5] [6] [9] [10].

The main complication reported by some studies is the diminution of visual acuity [4]. As in our study all patients had been submitted to CFC, there was a low pre-operative $\mathrm{VA}$, it was not possible to measure the results similarly.

As regards transitory adverse effects, there is frequently hyphaema $<3 \%$ [1] [2] [9]. In our study, we found a greater prevalence of neovascular glaucoma in our sample in comparison with the cited studies.

Concerning the limitations inherent to the design of the study it is important to note that as it is an observational study with a retrospective collection of data, it is not possible to control the number of observers and the bias associated to the inter- and intra-individual variability of the measurements of IOP or of the performance of the procedure itself. Apart from that we would have liked to study the scale of pain, which due to the internal validation of the study, were not possible to include.

\section{Conclusion}

The results presented in this study are in line with the current literature [1] [3] [6] [8] [9] [10] and so one may conclude that CFC is a simple, effective and safe procedure, which permits an improvement in the quality of life of patients with refractory glaucoma through the diminution of the number of anti-glaucoma drugs, the sustained reduction of IOP and the resolution of painful symptomology.

\section{References}

[1] Osman, E.A., Al-Muammar, A., Al-Mezaine, H. and Al-Obeidan, S.A. (2010) Controlled Cyclophotocoagulation with Diode Laser in Refractory Glaucoma and Long Term Follow up at King Abdulaziz University Hospital, Riyadh. Saudi Journal of Opthalmology, 24, 9-13. https://doi.org/10.1016/j.sjopt.2009.12.002

[2] Lima, V.C.W., Mello, P.A.A. and Junior, J.A.P. (2003) Ciclofotocoagulação com la- 
ser díodo em glaucoma refratário, resultado a longo prazo. Arquivos Brasileiros de Oftalmologia, 66. https://doi.org/10.1590/s0004-27492003000400010

[3] Hennis, H.L. and Stewart, W.C. (1992) Semiconductor Diode Laser Transscleral Cyclophotocoagulation in Patients with Glaucoma. American Journal of Ophthalmology, 113, 81-85. https://doi.org/10.1016/S0002-9394(14)75758-7

[4] Kyoko, I. (2013) Update on Results and Complications of Cyclophotocoagulations. Current Opinion in Ophthalmology, 24 102-110. https://doi.org/10.1097/ICU.0b013e32835d9335

[5] Ness, P.J., Khaimi, M.A., Feldman, R.M., Tabet, R., et al. (2012) Intermediate Term Safety and Efficacy of Transscleral Cyclophotocoagulation after Tube Shunt Failure. Journal of Glaucoma, 21, 83-88.

[6] Feldman, R.M., El-Harazi, S.M., LoRusso, F.J., et al. (1997) Histopatologic Findings Following Contact Transscleral Semiconductor Diode Laser Cyclophotocoagulation in Human Eye. Journal of Glaucoma, 6, 139-140. https://doi.org/10.1097/00061198-199704000-00011

[7] McKelvie, P.A. and Walland, M.J. (2002) Pathology of Cyclodiode Laser: A séRies of Nine Enucleated Eyes. British Journal of Ophthalmology, 86, 381-386. https://doi.org/10.1136/bjo.86.4.381

[8] Meyer, J.J. and Lawrence, S.D. (2012) What's New in Laser Treatment for Glaucoma? Current Opinion in Ophthalmology, 23, 111-117. https://doi.org/10.1097/ICU.0b013e32834f1887

[9] Frezzotti, P., Mittica, V., Martone, G., et al. (2010) Longterm Follow-Up of Diode Laser Trasscleral Cyclophotocoagulation in Treatment of Refratory Glaucoma. Acta Ophthalmologica, 88, 150-155. https://doi.org/10.1111/j.1755-3768.2008.01354.x

[10] Chen, T. and Chhabra, S. (2014) Diodo Laser Transscleral Cyclophotocoagulation. Digital Journal of Oftalmology.

\section{Submit or recommend next manuscript to SCIRP and we will provide best service for you:}

Accepting pre-submission inquiries through Email, Facebook, LinkedIn, Twitter, etc. A wide selection of journals (inclusive of 9 subjects, more than 200 journals)

Providing 24-hour high-quality service

User-friendly online submission system

Fair and swift peer-review system

Efficient typesetting and proofreading procedure

Display of the result of downloads and visits, as well as the number of cited articles

Maximum dissemination of your research work

Submit your manuscript at: http://papersubmission.scirp.org/

Or contact ojoph@scirp.org 